\title{
Study of the Enhancement of Dipolar Resonant Excitation by Linear Ion Trap Simulations
}

\author{
Sheldon M. Williams* and K. W. Michael Siu \\ Department of Chemistry and Center for Research in Mass Spectrometry, York University, Toronto, Ontario, \\ Canada
}

\author{
Frank A. Londry and Vladimir I. Baranov \\ MDS SCIEX, Concord, Ontario, Canada
}

Resolution improvements in dipolar resonant excitation have been examined in a round-rod quadrupolar collision cell for values of the Mathieu characteristic exponent $\beta$ equal to $n / p$, where $n$ and $m$ are small integers (prime $\beta$ values) versus other $\beta$ values where $n$ and $p$ are not small (ordinary $\beta$ values). The trajectories of ions moving in the time-varying electric fields of a quadrupole with and without buffer-gas molecules were calculated to determine the relationship of prime and ordinary $\beta$ values to frequency resolution for resonant ion excitation and ejection. For prime $\beta$ values, the ion trajectory in the hyperbolic quadrupole field will be exactly periodic with a period of at most $4 \pi p / \Omega$, where $\Omega$ is the angular frequency of the main drive radio-frequency (RF) potential. Ion trajectory simulations with prime $\beta$ versus ordinary $\beta$ values show that the motion of ions with prime $\beta$ values have simpler trajectories of shorter periods. Frequency response profiles (FRPs) for round-rod quadrupoles at zero pressure show that dipolar resonant excitations with prime $\beta$ values exhibit significantly narrower bandwidths than those with ordinary $\beta$ values. Simulations show that at 0.05 to 0.8 mTorr of nitrogen, it is possible to reduce the FRP bandwidth by $20 \%$ (measured at $50 \%$ depth). (J Am Soc Mass Spectrom 2007, 18, 578-587) @ 2007 American Society for Mass Spectrometry

$\mathrm{I}$ a 3D quadrupole ion trap (Paul trap) mass spectrometer, ion storage and confinement are accomplished by the application of a time-dependent, radio-frequency (RF) electric field [1]. By scanning the RF voltage, trapped ions may be ejected one $m / z$ ratio at a time out of the confining electric field for detection. While the ions are confined, ion-molecule reactions may be carried out. Additionally, the kinetic energy of the trapped ions can be increased by applying a dipolar AC potential that is resonant with the secular frequency of the motion of ions $\left(\omega_{0}\right)$ of one or more $\mathrm{m} / \mathrm{z}$ values. This increased kinetic energy can be used to selectively eject the ions from the trapping field, or to add internal energy to the confined ions through collisions with a neutral gas added to the trap. This dipolar resonant excitation has been a common method for performing mass-selective ejection and fragmentation of ions confined in Paul traps and, more recently, in linear ion traps [2-4]. The theory of instability space, as it relates to auxiliary AC excitation in a linear and 3D quadrupole trap for quadrupolar resonant excitation,

Published online December 22, 2006

Address reprint requests to Vladimir I. Baranov, University of Toronto, IBBME, 4 Taddlecreek Road, Room 407, Toronto, Ontario M5S 3G9 Canada. E-mail: vladimir.baranov@utoronto.ca

* Current address: Washburn University, Topeka, Kansas, USA. has been addressed [5-7]. The trajectories of ions in a linear ion trap with dipolar auxiliary excitation have been examined by Franzen [8]; those for hyperbolic and circular quadrupole rods with and without collision gas by Collings et al. [2].

In tandem mass spectrometry (MS/MS), resolution of the product ions is sometimes crucial as many fragments have similar $m / z$ values [9-15]. Unambiguous product-ion assignment often requires better than unit-mass resolution to resolve "isobaric" interferences, which are particularly acute when the analyte is the minor component. Two factors that degrade $m / z$ resolution after dipolar resonance excitation are increasing collisions with buffer-gas molecules [2] and increasing dipolar voltage amplitude [16]. One method to increase resolution is to excite the ions in such a way to reduce the frequency composition and the periodicity of the ions' motion in the electric field. In the present study, we used simulation software to determine the trajectories of ions [17] moving in a time-varying electric field and colliding with buffer-gas molecules in a linear quadrupole reaction cell with the instrumentally common and practical round-rod geometry.

It is a common misconception that an ion trajectory within the quadrupolar field is necessarily aperiodic. As we will show below, provided that the Mathieu char- 
acteristic exponent $\beta$ is a fraction $n / p$ of integers, then the ion trajectory is exactly periodic with a period of at most $4 \pi p / \Omega$, where $\Omega$ is the RF angular frequency. Under the influence of a resonant dipolar AC field, such an ion, with a $\beta$ value of $n / p$ ratio, where $n$ and $p=1,2$, $3, \ldots 7$ and here defined as having a prime $\beta$ value, should experience a faster linear increase in its radial amplitude than an ion with a $\beta$ value comprising $n$ and $p$ values that are not small integers, i.e., having an ordinary $\beta$ value. Practically, the integer range for $p$ is limited to one to seven, inclusive, to clearly observe the aforementioned improvements in resolution, ejection time, and simplicity of the ion trajectory. The scope of this study is limited to the first stability region, so $\beta<$ 1 and $n<p$. For the sake of convenience, the ion trajectory resulting from a prime $\beta$ value is referred to here as a prime trajectory; similarly, that resulting from an ordinary $\beta$ value as an ordinary trajectory. Operation at prime $\beta$ values thus allows for shorter ion ejection times and fewer ion-molecule collisions, which normally decrease resolution. The simpler ion trajectory should also narrow the bandwidth of the frequency response profile (FRP), which is a plot of the ion ejection time versus the auxiliary AC angular frequency, and increases resolution. This strategy for resolution enhancement should be particularly effective when the ion ejection rate is faster than the ion-neutral collision rate. The instrument in which this condition can readily be met is the linear ion trap [2].

Most 2D quadrupoles employ circular as opposed to hyperbolic rods. Here, resolution considerations are even more germane, as deviation from the hyperbolic geometry creates additional higher-order electric fields, i.e., octapolar, etc. [2, 18-20]. Some of these higherorder fields are off-resonant with the ion's secular motion, while the ion is stable in the quadrupolar field. These off-resonant higher-order fields increase in strength radially. As an ion becomes excited during resonance with the quadrupolar field and its trajectory envelope expands and approaches the quadrupole rods, it loses energy in the region proximal to the rods as a result of strengthening higher-order fields. This loss of energy leads to a decrease of the ion's trajectory envelope. Thus, the resultant effect is that the ion's trajectory is roughly periodic with increasing and decreasing radii until sufficient resonant energy is added to accelerate it out of the electric field [2] (otherwise, an ion in such a situation can theoretically be trapped for an indefinite period). However, increasing the AC potential reduces frequency resolution, thus it would be helpful if the loss of resolution brought about by increasing the dipolar excitation energy could be offset by a resolution enhancement method. Circular rods also introduce greater complexity into the frequency power spectrum of the ion trajectory: additional frequencies may overlap with the fundamental secular frequency that may counteract any resolution enhancement methods. These additional frequencies occur as harmonics of the fundamental secular frequency and frequencies induced by the aux- iliary AC for resonant excitation. Therefore, it is also critical to investigate the occurrence of resonances that are not due to the secular frequency of the ion by creating plots of ejection time versus frequency for prime $\beta$ values and resolution (FRP bandwidth) versus $\beta$ value. To the best of our knowledge, prior studies of nonlinear fields, dipolar AC excitation induced resonances, or other resonances not due to harmonics of an ion's fundamental secular frequency $\left(\omega_{\mathrm{o}}\right)$ for linear quadrupoles were first investigated in 2003 [2]. Previously, extensive theoretical and simulation studies of fields and resonance effects for Paul ion traps were published in two series of articles between 1989 and 1994 [8, 21-29]. Two of these articles discuss dipolar resonant excitation $[8,28]$. Enhancement in resolution of dipolar excitation can be brought about by increasing the $\beta$ value via an increase in the amplitude of the main RF voltage. The bandwidth of an FRP at $\beta=2 / 3$ is about half that of $\beta=1 / 4$. However, increasing the $\beta$ value can have deleterious effects on the minimum $\mathrm{m} / \mathrm{z}$ value of ions trapped; experimentally, operating at a higher $\beta$ value may lead to problems pertaining to ion storage, due to larger overall radii and higher secular frequencies of ions. As described in two manuscripts by Londry and Hager and Stott et al., a method was developed for increasing FRP resolution by varying the DC potential difference between the quadrupole rods and exit lens of a linear ion trap as a function of ion mass [17, 20].

Konenkov made one of the first applications of prime periodic trajectories in mass spectrometry (personal communication with Nikolai Konenkov, 1998). He postulated that ions of a single $m / z$ value and appropriate axial velocity entering the inlet aperture of a quadrupole, and having a prime periodic trajectory, can selectively exit the quadrupole through the exit aperture excluding ions of similar $\mathrm{m} / \mathrm{z}$ values.

Though practical restrictions on exit aperture diameter and axial velocity distribution limit the improvements in resolution, the principle is still applicable to explaining various facets of ion behavior in a quadrupole, such as the peak-splitting phenomenon described by Du et al. [30]. Another early application of prime periodic trajectories was made by Londry and March that illustrates the resolution enhancements attainable using prime $\beta$ values [31]. Experimental results showed that adjusting the phase of the auxiliary AC potential with respect to the main RF potential can improve the frequency resolution of an ion when it has a prime $\beta_{z}$ value versus an ordinary $\beta_{z}$ value. Resulting improvement in $m / z$ resolution, i.e., decrease in $\mathrm{m} / \mathrm{z}$ peak-width, was up to $20 \%$.

In short, previous studies show that judicious choices of $\beta$ values and auxiliary dipole potentials, will maximize resonant resolution. Here, we report results of a systematic examination of resolution enhancement using prime $\beta$ values via computer simulations of the ion trajectory in a circular-rod quadrupole. Studies on deviations from ideality in $\beta$ versus frequency bandwidth and on FRPs versus the auxiliary AC angular frequency are also reported. 


\section{Methods}

A custom simulation package, Sx32 version 11.0, developed at MDS SCIEX by one of us (FAL) was employed for ion trajectory calculations. Simulations were performed on a Dell Dimension 8300 PC with dual 3.0 GHz Pentium 4 processors. The Sx package uses the Excel (Microsoft Office 2000) graphical user interface (GUI) via Virtual Basic-programmed macros to interface input parameters with a $\mathrm{C}^{++}$program, $\mathrm{R} \times 32$, that calculates trajectories; the output parameter values are then displayed with the Excel GUI (See [17]). Each ion trajectory is calculated incrementally, by integrating the secondorder differential equation of motion, twenty times each $\mathrm{RF}$ cycle for $10 \mathrm{~ms}$, or until the ion strikes a quadrupole rod. For a singly charged positive ion,

$$
\frac{d^{2} \vec{r}}{d t^{2}}=\frac{e}{m} \vec{E}
$$

where $\vec{r}$ is the position vector of the ion, $e$ is the electronic charge, $m$ is the mass of the ion, and $\vec{E}$ is the electric field. When necessary, several mathematically distinct fields are superimposed within a single trajectory calculation by determining the vector sum of the contributing forces. eq. 1 is integrated numerically using Richardson extrapolation and the Bulirsch-Stoer method with adaptive size and error control [32]. The electric field is obtained by interpolating tabulated numerical solutions to the Laplace equation, as no analytical expression exists for the electric field created by a circular-rod quadrupole. The numerical solutions have previously been obtained using a relaxation solver developed in-house. Quadrupole rods of hyperbolic or circular geometry can be specified for either $20.0 \mathrm{~cm}$ or infinite length. Other geometries are also available. Dipolar and quadrupolar resonant excitation functions originating from hyperbolic or circular rods are also available.

For the present study, the quadrupole was made infinitely long to initially eliminate ions interacting with the collision cell's fringing fields. Dipolar excitation was applied to the quadrupolar rods. The shortest distance between the quadrupolar axis and the rods, $r_{0}$, was $4.695 \mathrm{~mm}$ and the radius of the circular rods, $r_{\mathrm{r}}$, was 4.17 $\mathrm{mm}$. The $\beta$ parameter was used to determine the corresponding secular frequency of the ion motion. FRPs were plotted by varying the Mathieu equation's $q$ stability parameter ( $a=0$, see also the Theory section), and determining the duration of an ion of $609 \mathrm{Th}$ (protonated reserpine) in the quadrupole, while a dipolar AC field was applied between one pair of opposing rods at a frequency resonant with the $609 \mathrm{Th}$ ion. The FRPs were determined by averaging the ejection times of twenty ions with different, randomly chosen $x, y$, and $z$-coordinate velocity values at $q$-parameter values in intervals of 0.0001 to 0.0003 , depending on the range of q-parameter values needed to describe the entire FRP peak. To measure the bandwidth for each FRP, the profile data points were least-squares fitted with a sixth-order polynomial, and the full width at half depth of the profile was determined. For the plotting of frequency bandwidth versus $\beta$, FRPs were determined at AC frequencies corresponding to several $\beta$ values surrounding and including a prime $\beta$ value. For the data presented in this manuscript, the q-parameter values have been converted to frequencies.

\section{Theory}

In most cases, the ion trajectory in the main quadrupole RF field has no apparent periodicity. Despite the welldefined set of frequencies in the power spectrum [33], complicated phase shifts and dependence on initial conditions make trajectories aperiodic. However, this is not applicable to all cases. From Floquet's theorem [34], it follows that, when the Mathieu characteristic exponent $\beta(a, q)$ [34] is a fraction $n / p$ of integers, the solution to the Mathieu equation

$$
\frac{d^{2} x}{d \xi^{2}}+(a-2 q \cos (2 \xi)) x=0
$$

is periodic and has a period of at most $4 \pi p / \Omega$. Here, $a$ and $q$ are dimensionless parameters and $\zeta$ is dimensionless time. For the particular case of the RF-driven two-dimensional quadrupolar field, we have $\frac{4 e U}{m \Omega^{2} r_{0}^{2}}=$ $a, \frac{2 e V}{m \Omega^{2} r_{0}^{2}}=q$ and $\xi=\frac{t \Omega}{2}$. The quadrupolar potential can be expressed as a combination of two terms having DC $(U)$ and $R F(V)$ trapping potentials:

$$
\Phi=\frac{\left(x^{2}-y^{2}\right)}{2 r_{0}^{2}}(U-V \cos (t \Omega))
$$

where $r_{0}$ is the rod-to-axis distance as defined above and $\Omega$ is the main RF angular frequency.

Let us investigate the property of the solution to the Mathieu equation in detail:

$$
x(\xi)=C_{1} C(a, q, \xi)+C_{2} S(a, q, \xi)
$$

$C(a, q, \zeta)$ is the even Mathieu function with the real characteristic value $a$ and parameter $q$, and $S(a, q, \zeta)$ is the odd Mathieu function. $C_{1}$ and $C_{2}$ are the integration constants and depend only on the initial conditions, including the initial phase.

According to Floquet's theorem, there exist two solutions to the Mathieu equation of the form:

$$
x(\xi)=e^{i \beta(a, q) \xi} P(\xi) \text { and } x(-\xi)=e^{-i \beta(a, q) \xi} P(-\xi)
$$

(the even and odd Mathieu functions, respectively) where the characteristic exponent $\beta$ depends on $a$ and $q$ (used without variables for brevity), and $P(\xi)$ is a periodic function of the same period as that of the coefficients in the Mathieu equation (2), namely $2 \pi / \Omega$. 


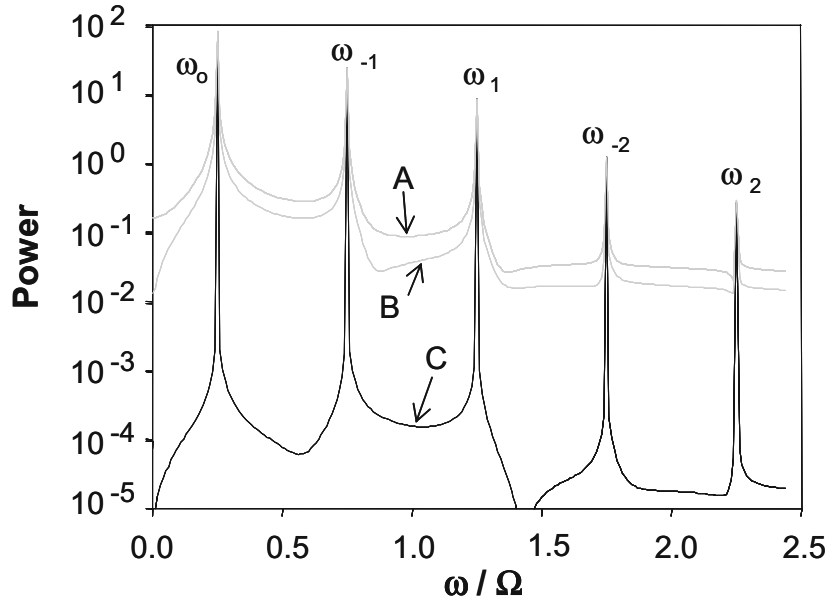

Figure 1. The power spectrum of the ion trajectory for prime $\beta$ value $1 / 2$ and ordinary $\beta$ values 0.502912 and 0.5009139 where $a=$ 0 and (a) $q=0.64, \beta=0.502912$; (b) $q=0.638, \beta=0.5009139$; (c) $q=0.639315, \beta=1 / 2$.

If the characteristic exponent $\beta$ is not an integer (not on a stability boundary), then the Floquet solutions are linearly independent and can be written in the form:

$$
x(\xi)=\sum_{k=-\infty}^{\infty} c_{2 k} e^{i(\beta+2 k) \xi}
$$

which has a Fourier transform with respect to $\omega$ of the form:

$$
\begin{gathered}
F(x(t \Omega / 2), \omega)=F\left(\sum_{k=-\infty}^{\infty} c_{2 k} e^{i(\beta+2 k) \frac{t \Omega}{2}}, \omega\right) \\
=\sum_{k=-\infty}^{\infty} c_{2 k} 2 \sqrt{2 \pi} \delta((\beta+2 k) \Omega+2 \omega)
\end{gathered}
$$

Here, $\delta(\mathrm{x})$ represents the Dirac delta function and $\xi$ is replaced by $t \Omega / 2$. Therefore, Fourier coefficients are non-zero only for $k=0, \pm 1, \pm 2, \ldots$, , which defines the fundamental secular frequency of the ion's trajectory,

$$
\omega_{0}=\Omega \frac{\beta}{2} \text { (macro motion) }
$$

and the harmonics,

$$
\omega_{k}=\Omega\left|k+\frac{\beta}{2}\right|, k= \pm 1, \pm 2, \ldots \text { (micro motions). }
$$

The ion motion in the quadrupole RF field can be considered as a superposition of macro and micro motions. Macro and micro motions contribute to the ion trajectory according to their amplitudes. Notice that the RF driving frequency $\Omega$ is not present in the power spectrum of the ion trajectory (see eq 7 and Figure 1).
Therefore, from eq 6 it follows that if $\beta$ is a rational fraction $n / p$, then the solution of the Mathieu equation is periodic and has a period of at most $4 \pi p / \Omega$. Therefore, for a periodic solution (which corresponds to a periodic trajectory), there is a simple relationship between RF driving frequency $\Omega$ and resonance frequency $\omega_{k}$ :

$$
\omega_{0}=\frac{n \Omega}{2 p} \text { and } \omega_{k}=\Omega\left|k+\frac{n}{2 p}\right|, k= \pm 1, \pm 2, \ldots
$$

where $n$ and $p$ are the numerator and denominator of a rational fraction $\beta=n / p$, respectively.

One should expect that, for the prime trajectory (integer values: $n \geq 1, p>n$ ), the power spectrum should be well-defined with sharp dominant frequencies $\omega_{0}$ and $\omega_{k}$. For prime $\beta$ values, the ion trajectory is exactly periodic: the smaller the integers, the shorter the trajectory period. The entire period of the trajectories for $\beta=1 / 3$ or $2 / 3$ is at most completed in $12 \pi / \Omega$, while that with ordinary $\beta$ values is much longer. For instance, for $\beta=0.292566\left(n / p=292,566 / 10^{6}\right)$, it is at least $4 \cdot 10^{6} \pi / \Omega$.

In the power spectra for $\beta=1 / 2$, the bandwidth of the ion's fundamental secular frequency is far narrower than those for ordinary $\beta$ values, such as 0.502912 and $0.5009139\left(n / p=502,912 / 10^{6}\right.$ and $5009,139 / 10^{7}$, respectively). In addition, the relative intensity of the fundamental secular frequency, $\omega_{0}$, compared with the frequency background, is several orders of magnitude greater for the prime $\beta$ value than those for the ordinary $\beta$ values. The same is true for $\omega_{k}$. This decreased bandwidth and increased relative intensity should result in improved resonance resolution with an AC frequency applied to the quadrupole. In terms of Fourier transformation, this improvement in resolution occurs because an ion can complete more cycles within a given time interval. The motion of a pendulum can serve as a much simplified analogy to the radial motion of an ion in the quadrupole field. Just as in the case of a pendulum that has a force applied to it once per period synchronously with the pendulum's motion, the shorter the period, the faster will the height of the pendulum swing increase.

Let us consider a cosine waveform for dipolar excitation in the quadrupolar field. The equation of motion should be (assuming that the excitation is along the $x$ axis):

$$
\frac{e}{r_{0}^{2}}(U-V \cos (t \Omega)) x(t)+m \ddot{x}(t)=\frac{e A}{r_{0}} \cos \left(t \omega_{A C}+\alpha\right)
$$

Here $\alpha$ is the phase shift between the AC (dipolar excitation) and the RF, and $A$ is $\mathrm{AC}$ amplitude. The determination of the phase shift can be eliminated by employing the trigonometric equality:

$$
\begin{aligned}
& A \cos \left(t \omega_{A C}+\alpha\right)=A \cos (\alpha) \cos \left(t \omega_{A C}\right) \\
& \quad-A \sin (\alpha) \sin \left(t \omega_{A C}\right)=A_{1} \sin \left(t \omega_{A C}\right)+A_{2} \cos \left(t \omega_{A C}\right)
\end{aligned}
$$


Therefore, the solution of eq 9 can be presented as a superposition of the transient eq 4 and "forced" solutions:

$$
\begin{aligned}
x(t) & =C_{1} C(a, q, t \Omega / 2)+C_{2} S(a, q, t \Omega / 2) \\
& +K\left[C ( a , q , t \Omega / 2 ) \int _ { 0 } ^ { t } \left(A_{1} \sin \left(\tau \omega_{A C}\right)\right.\right. \\
& \left.+A_{2} \cos \left(\tau \omega_{A C}\right)\right) S(a, q, t \Omega / 2) d \tau \\
& +S(a, q, t \Omega / 2) \int_{0}^{t}\left(A_{1} \sin \left(\tau \omega_{A C}\right)\right. \\
& \left.\left.+A_{2} \cos \left(\tau \omega_{A C}\right)\right) C(a, q, t \Omega / 2) d \tau\right]
\end{aligned}
$$

The new integration constant $K$ can be determined easily, but this will not be elaborated here for brevity. The variable $\tau$ is an integration variable with an identity that is dependent on the upper limit of its corresponding integral. The integrals in the solution eq 10 deserve a closer look. Integrals of this type are analogous to Fourier integrals (integrals for Fourier coefficients). For $\omega_{A C}=\omega_{0}=\frac{n \Omega}{2 p}$ and $t=4 \pi p / \Omega$ (over one period), the following relationships exist:

$$
\begin{aligned}
& \frac{2}{p \pi} \int_{0}^{p \pi} \cos \left(\frac{n \tau}{p}\right) C(a, q, \tau) d \tau \rightarrow 1 \\
& \frac{2}{p \pi} \int_{0}^{p \pi} \sin \left(\frac{n \tau}{p}\right) C(a, q, \tau) d \tau \rightarrow 0 \\
& \frac{2}{p \pi} \int_{0}^{p \pi} \cos \left(\frac{n \tau}{p}\right) S(a, q, \tau) d \tau \rightarrow 0 \\
& \frac{2}{p \pi} \int_{0}^{p \pi} \sin \left(\frac{n \tau}{p}\right) S(a, q, \tau) d \tau \rightarrow 1
\end{aligned}
$$

Integrals that are close to zero over one period will not contribute much over a long time interval; the integrals that are close to unity will contribute in every period and the contribution will accumulate proportionally to the time interval and the trajectory period. This analysis is one more proof of eq 7 with respect to resonant frequency $\omega_{A C}$. With these points in mind, solution eq 10 can be simplified:

$$
\begin{aligned}
x(t) & =C_{1} C(a, q, t \Omega / 2)+C_{2} S(a, q, t \Omega / 2) \\
& +K\left[A_{1} C(a, q, t \Omega / 2) \int_{0}^{t} \sin \left(\tau \omega_{A C}\right) S(a, q, t \Omega / 2) d \tau\right. \\
& \left.+A_{2} S(a, q, t \Omega / 2) \int_{0}^{t} \cos \left(\tau \omega_{A C}\right) C(a, q, t \Omega / 2) d \tau\right]
\end{aligned}
$$

Consider, for example, the integral

$$
I_{13}(a, q, N)=\frac{2}{N p \pi} \int_{0}^{N p \pi} \sin (\gamma \tau) S(a, q, \tau) d \tau,
$$
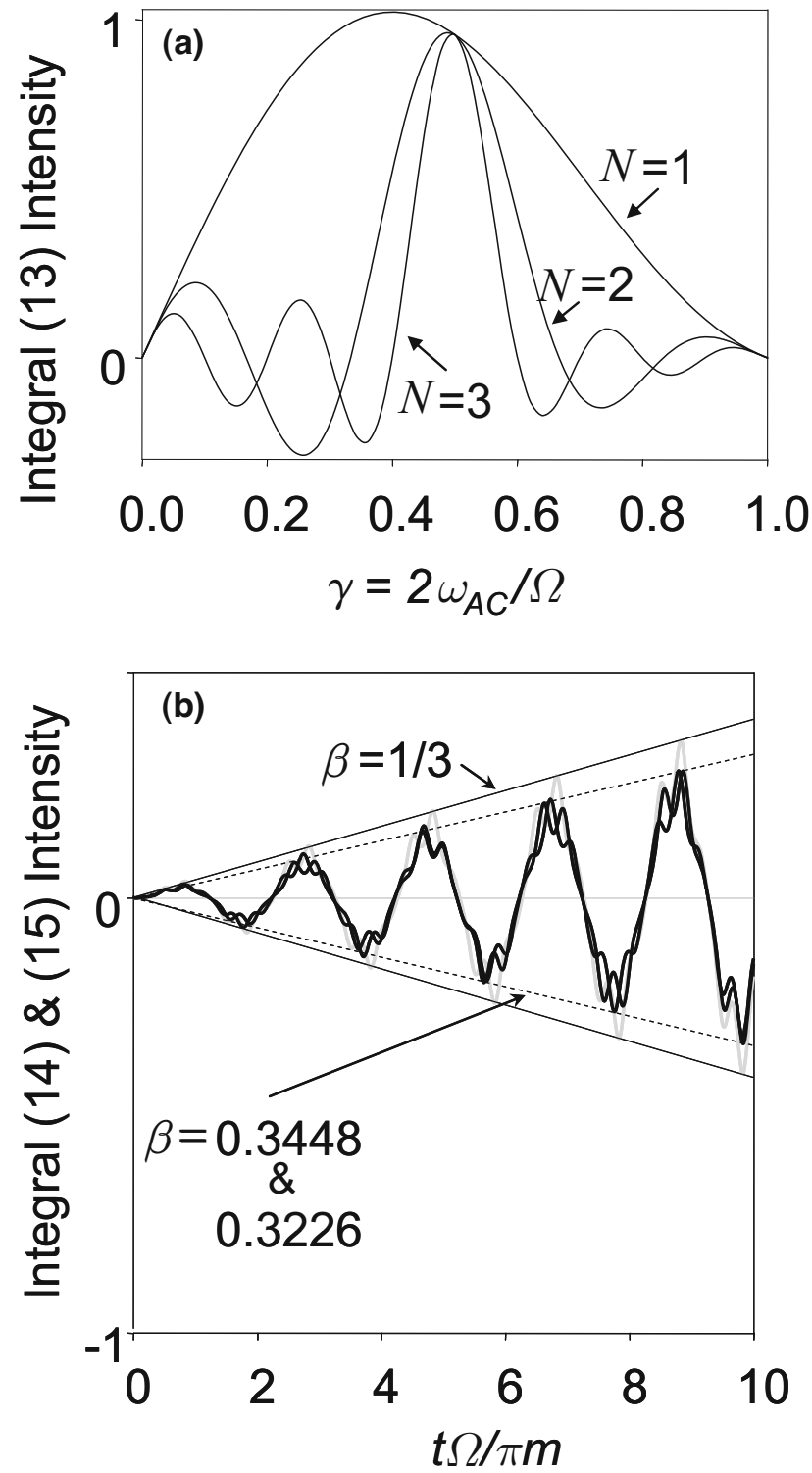

Figure 2. Normalized integral intensity for: (a) eq 13 versus the frequency interval $\gamma=0$ to 1 . Plot describes the frequency response profile for resonant excitation at $\beta=0.5$ for number of exact ion trajectories completed, $N$; (b) eqs 14 and 15 versus $t \Omega / \pi p$. Plot describes the increasing radial amplitude of an ion with time experiencing resonant excitation at the prime $\beta$ value of $1 / 3$ and two similar ordinary $\beta$ values, $0.3448\left(n / p=3448 / 10^{4}\right)$ and $0.3226\left(n / p=3226 / 10^{4}\right)$.

which is on interval $\gamma=\frac{2 \omega_{A C}}{\Omega} \in[0,1]$ depends on the number of periods $N$ of the prime trajectory as presented in Figure 2a for $\beta=0.5(n / p=1 / 2, q=0.639315)$. It will take several RF periods for integral (eq13) to achieve a sharp resonance and, therefore, a shorter period should allow faster ion ejection. Therefore, for resonant conditions and prime trajectories the following integrals, (eq 14) and (eq 15), are virtually indistinguishable over the time interval of several periods of the prime trajectory: 


$$
\begin{aligned}
& I_{14}(a, q, t \Omega / 2)=C(a, q, t \Omega / 2) \int_{0}^{t}\left(A_{1} \sin \left(\tau \omega_{A C}\right)\right. \\
& \left.\quad+A_{2} \cos \left(\tau \omega_{A C}\right)\right) S(a, q, \tau \Omega / 2) d \tau \\
& +S(a, q, t \Omega / 2) \int_{0}^{t}\left(A_{1} \sin \left(\tau \omega_{A C}\right)\right. \\
& \left.\quad+A_{2} \cos \left(\tau \omega_{A C}\right)\right) C(a, q, \tau \Omega / 2) d \tau \\
& I_{15}(a, q, t \Omega / 2) \\
& \quad=A_{1} C(a, q, t \Omega / 2) \int_{0}^{t} \sin \left(\tau \omega_{A C}\right) S(a, q, \tau \Omega / 2) d \tau \\
& \quad+A_{2} S(a, q, t \Omega / 2) \int_{0}^{t} \cos \left(\tau \omega_{A C}\right) C(a, q, \tau \Omega / 2) d \tau \\
& =\frac{t}{2}\left[A_{1} C(a, q, t \Omega / 2)+A_{2} S(a, q, t \Omega / 2)\right]
\end{aligned}
$$

and are presented in Figure $2 b$.

From integrals (eq 11), (eq 14), and (eq 15) it follows that the amplitude of resonant trajectory increases linearly with the AC amplitude and time (or number of completed periods):

$$
\begin{aligned}
& x(t)=C_{1}^{\prime} C(a, q, t \Omega / 2) \\
& +C_{2}^{\prime} S(a, q, t \Omega / 2),\left\{\begin{array}{l}
C_{1}^{\prime}=C_{1}+\frac{t}{2} K A_{1} \\
C_{2}^{\prime}=C_{2}+\frac{t}{2} K A_{2}
\end{array}\right.
\end{aligned}
$$

The prime trajectory has a similar dependence on time as any other trajectory, but its amplitude grows faster because the trajectory period is shorter.

Although an ideal quadrupole field is convenient for theoretical consideration and the theoretical background offers us indispensable insight into resonant effects in the quadrupole field, practical implications should be evaluated employing a realistic circular-rod quadrupole assembly. The theory presented in this section applies strictly to the ideal quadrupolar fields and dipolar AC excitation created by ideal hyperbolic rods. Since quadrupoles with round rods are commercially available and are more common than hyperbolic rods, the Sx32 simulation package was used to explore the application of the theory presented here to quadrupoles with round-rod geometry.

\section{Results and Discussion}

To demonstrate the generally applicability of improving frequency resolution for resonant excitation and ejection of ions at prime $\beta$ values, the variation in the bandwidth of the FRP for the fundamental secular frequency of the 609 Th ion was determined in an interval around three prime $\beta$ values. For reference, a
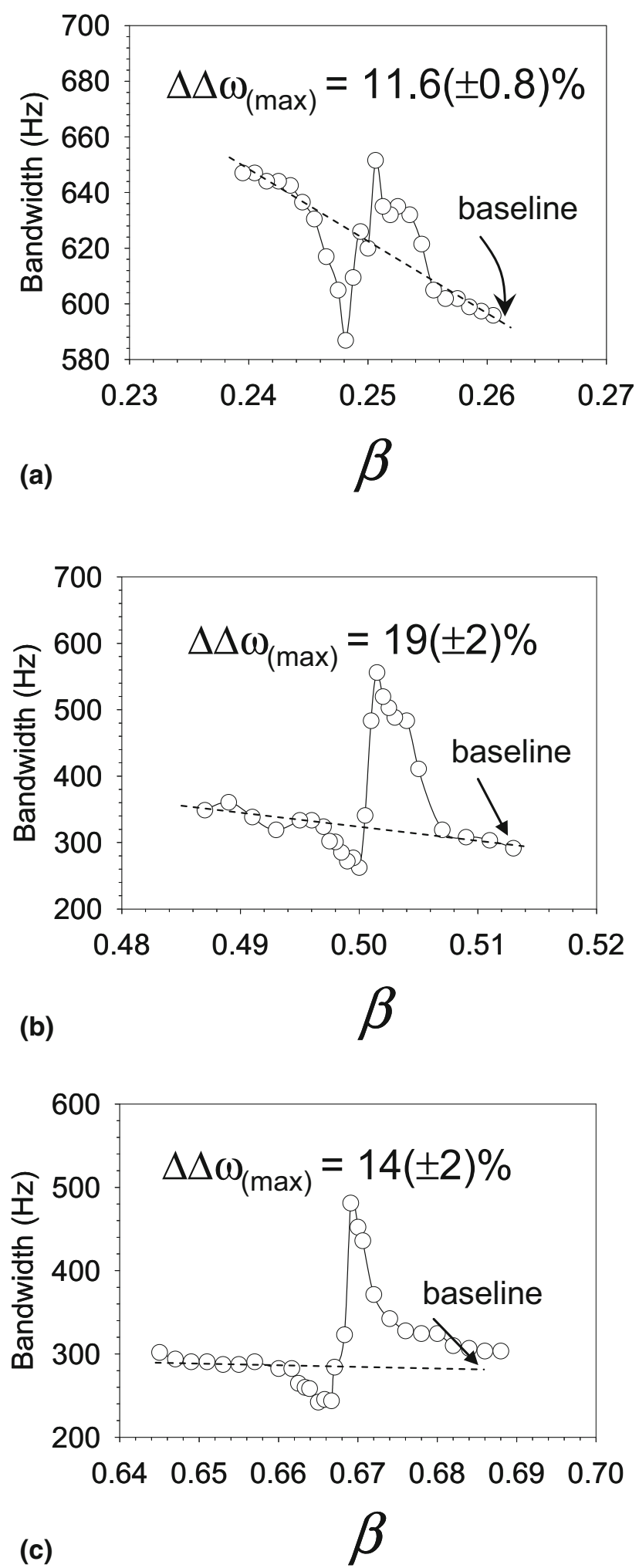

Figure 3. Frequency bandwidth versus $\beta_{\mathrm{x}}$ for (a) $\beta_{\mathrm{x}}=0.25 \pm$ 0.01 , (b) $0.5 \pm 0.013$, and (c) $0.666 \pm 0.022$, quadrupole cell with round rods at zero pressure and $\mathrm{V}_{\mathrm{ac}}=0.13 \mathrm{~V}$.

peak width of 1.0 Th corresponds to a $100 \mathrm{~Hz}$ bandwidth for the 609 Th ion. Figure 3a, b, and c show the bandwidths at half height of the FRPs for the 609 Th ion 
Table 1. Reductions in bandwidth $(\mathrm{Hz})$ for prime vs. ordinary $\beta$ values with varying AC voltage, measured at half depth of frequency response profile minimum for linear quadrupole cell with round rods and zero pressure

\begin{tabular}{lccc}
\hline & \multicolumn{3}{c}{$\beta$ values } \\
\cline { 2 - 4 } & $\beta=0.2500$ vs. & $\beta=0.3333$ vs & $\beta=0.5000$ vs. \\
$\mathrm{V}_{\mathrm{AC}}$ & $0.2531^{\mathrm{a}}$ & $0.3373^{\mathrm{b}}$ & $0.5027^{\mathrm{c}}$ \\
\hline \hline $0.5 \mathrm{~V}$ & $-16 \mathrm{~Hz}$ & $-25 \mathrm{~Hz}$ & $-24 \mathrm{~Hz}$ \\
$0.4 \mathrm{~V}$ & $-13 \mathrm{~Hz}$ & $-11 \mathrm{~Hz}$ & $-44 \mathrm{~Hz}$ \\
$0.3 \mathrm{~V}$ & $-6.3 \mathrm{~Hz}$ & $-11 \mathrm{~Hz}$ & $-36 \mathrm{~Hz}$ \\
\hline
\end{tabular}

${ }^{\mathrm{a}}$ For $\beta=0.2500, n / p=1 / 4$ and for $\beta=0.2531, n / p=2531 / 10^{5}$

${ }^{\mathrm{b}}$ For $\beta=0.3333, n / p=1 / 3$ and for $\beta=0.3373, n / p=3373 / 10^{5}$.

${ }^{c}$ For $\beta=0.5000, n / p=1 / 2$ and for $\beta=0.5027, n / p=5027 / 10^{5}$.

in a quadrupole cell with round rods and zero pressure around the prime $\beta$ values: $1 / 4,1 / 2$, and $2 / 3$. In each case, a significant decrease in bandwidth is observed that is centered at or very close to the prime $\beta$ value, known as "stability holes", showing an improvement in resonant frequency resolution of $11.6( \pm 0.8)$ to $19( \pm 2) \%$ compared with the baseline. The baseline was established by approximating a line through roughly linear portions of the data at the horizontal extrema of the measured $\beta$ values for that graph with uncertainty of approximately $\pm 5 \mathrm{~Hz}$. In some cases, the presence of the higher-order fields due to the round rounds creates "stability peaks or mountains", where the resolution is significantly worse compared with the bandwidth baseline, overlapping the stability holes. These significant increases and decreases in bandwidth provide valuable knowledge for optimizing resonance resolution. It is unlikely that the phase relationship between the $\mathrm{AC}$ and RF waveforms would affect the position or magnitude of the stability mountains, as phase shift has little significance at zero pressure. Rod shape and positioning, which have a large effect on the contributions of higher-order fields within the quadrupole cell, are likely to be the most important in determining the position and magnitude of stability holes and mountains.

Simulations of a more limited extent were conducted at higher AC voltages. Table 1 shows that significant reductions in bandwidth occur at higher $\mathrm{AC}$ voltages as well. Consequently, the improvements in resolution obtainable by performing resonant excitation and ejection of ions can be realized at increased AC voltages, which allows for faster ejection times. This feature can be especially important in the presence of collision gas. The variations in bandwidth reduction with AC voltage and prime $\beta$ value do not show any clear and general trends. Again, changes in the AC voltage may make significant alterations in the bandwidth versus $\beta$ plot topography.

In the next stage of the investigation, the effects of prime $\beta$ values on the resonant ejection of the 609 Th ion in the presence of nitrogen collision gas were determined. Figure 4 demonstrates how the combination of collision gas and round rods acts to increase the
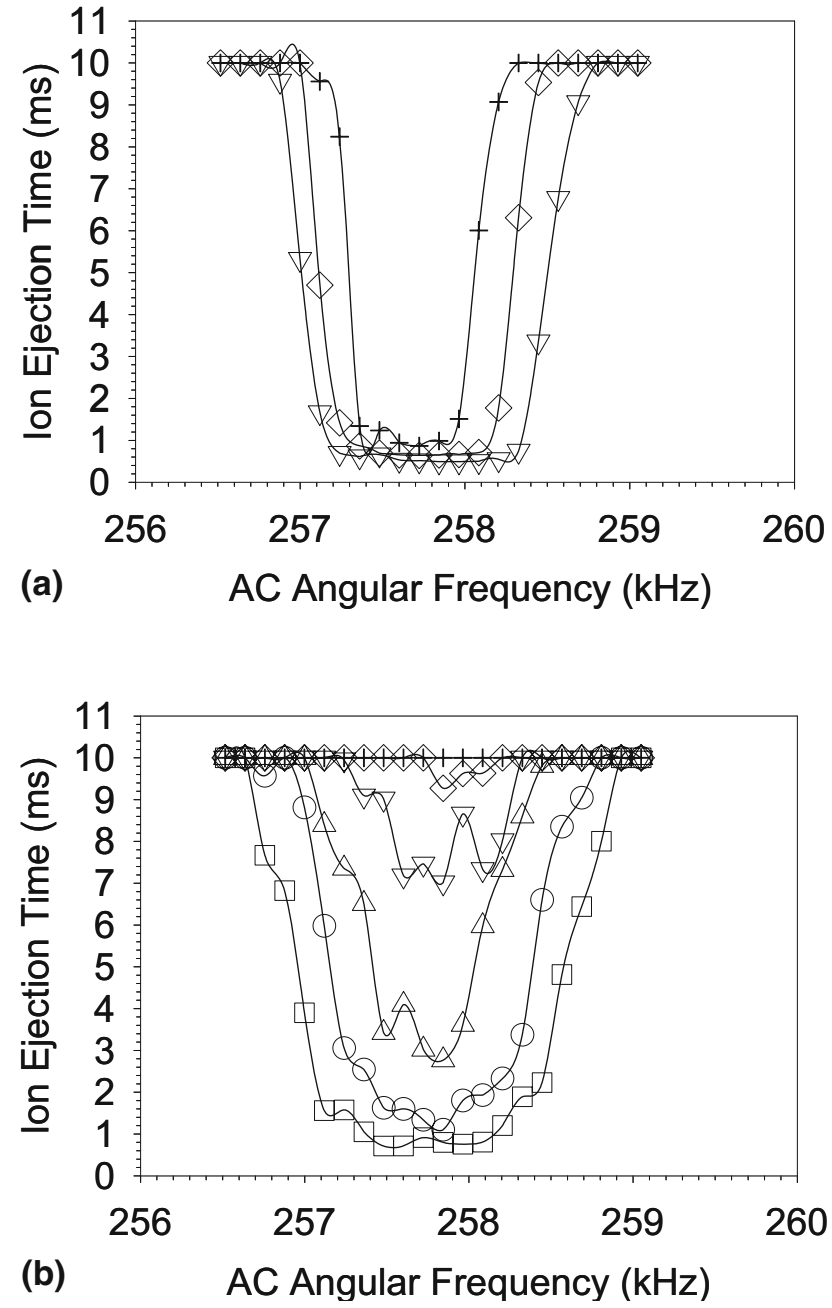

Figure 4. Effect of gas pressure on resolution and efficiency of resonant ejection. Frequency response profiles for $\beta=0.5027(n / p$ $\left.=5027 / 10^{4}\right)$, linear quadrupole with round rods, and $\mathrm{AC}$ voltages of $0.8(\square), 0.7(\bigcirc), 0.6(\triangle), 0.5(\nabla), 0.4(\diamond)$, and $0.3(+)$ volts. $(\mathbf{a}) 0.2$ mTorr nitrogen; (b) 0.8 mTorr nitrogen.

ejection time and the FRP bandwidth at 0.2 mTorr versus $0.8 \mathrm{mTorr}$. The increased pressure at $0.8 \mathrm{mTorr}$ greatly increases the ejection time for the 609 Th ion at $\mathrm{AC}$ voltages of $0.3,0.4$, and $0.5 \mathrm{~V}$. The $\mathrm{AC}$ voltage must be increased up to $0.8 \mathrm{~V}$ to re-attain an ejection time less than $1 \mathrm{~ms}$. Table 2 shows the reductions in bandwidth

Table 2. Reductions in bandwidth (Hz) for prime vs. ordinary $\beta$ values with varying pressure of nitrogen gas, measured at half depth of frequency response profile minimum for linear quadrupole cell with round rods

\begin{tabular}{llcc}
\hline Pressure $\mathrm{N}_{2}$ & $\mathrm{~V}_{\mathrm{ac}}(\mathrm{V})$ & $\begin{array}{c}\beta=0.25 \mathrm{vs} \\
0.2531^{\mathrm{a}}\end{array}$ & $\begin{array}{c}\beta=0.67 \mathrm{vs} \\
0.66167^{\mathrm{b}}\end{array}$ \\
\hline \hline $\mathrm{P}=0.00 \mathrm{mTorr}$ & 0.09 & -53.7 & -37.2 \\
0.05 mTorr & 0.1 & -17.1 & -31.1 \\
$0.10 \mathrm{~m}$ Torr & 0.12 & -1.9 & +6.9 \\
0.20 mTorr & 0.14 & -3.8 & 0 \\
\hline
\end{tabular}

${ }^{\mathrm{a}}$ For $\beta=0.25, n / p=1 / 4$ and for $\beta=0.2531, n / p=2531 / 1 \times 10^{5}$. ${ }^{\mathrm{b}}$ For $\beta=0.67, n / p=2 / 3$ and for $\beta=0.66167, n / p=66167 / 10^{6}$. 

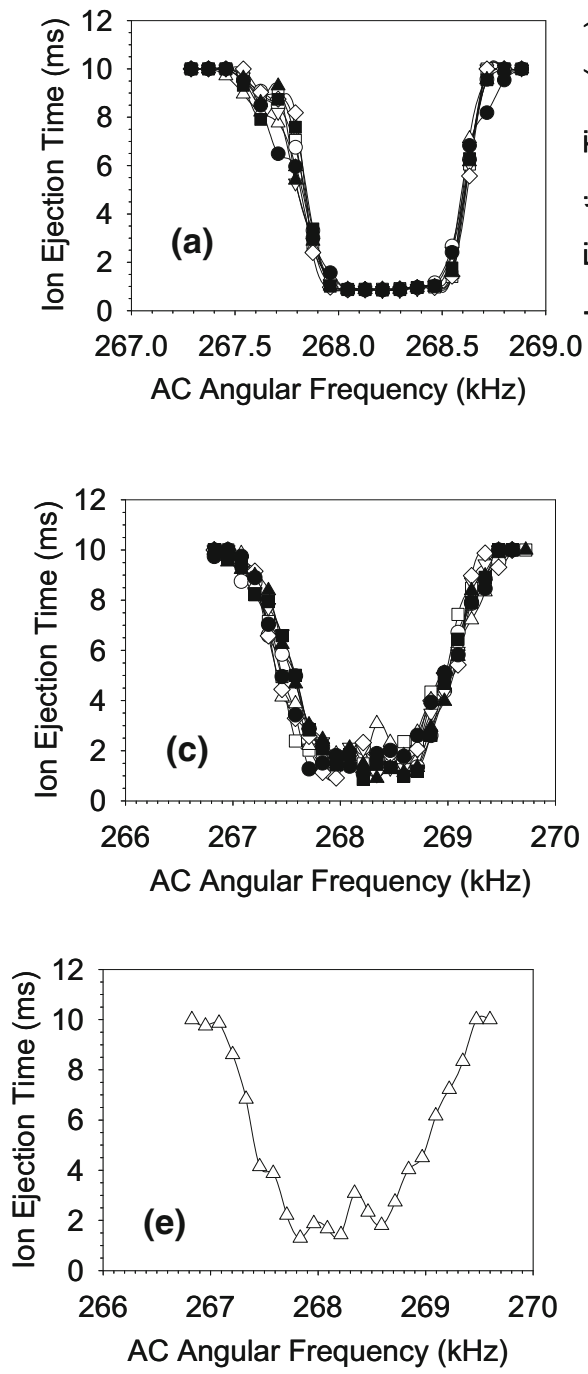
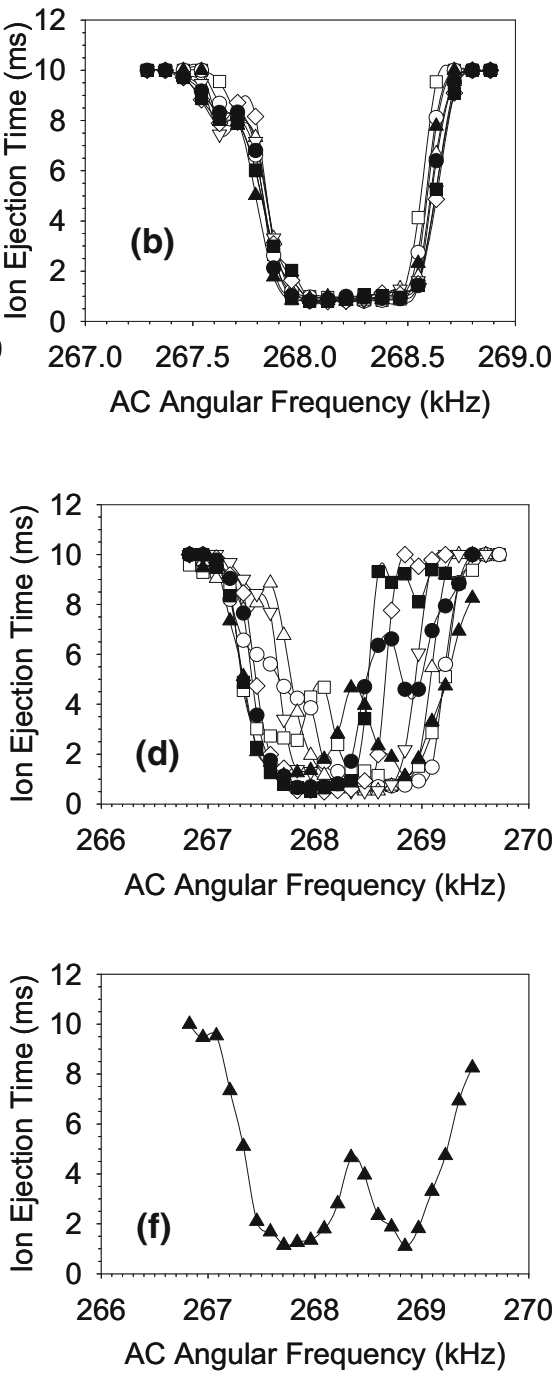

\begin{abstract}
Figure 5. Changes in FRP resolution with varying phase angles in degrees: $0(\boldsymbol{\square}), 45(\boldsymbol{O}), 90(\mathbf{\Delta}), 135$ $(\nabla), 180(\diamond), 225(\square), 270(\bigcirc)$, and $315(\triangle)$ (a) at zero pressure with initial AC phase, $\alpha$, independent of RF drive phase and (b) at zero pressure and initial AC phase, $\alpha^{*}$, locked relative to RF drive phase, or (c) the same as (a), but at 0.8 mTorr nitrogen gas and (d) the same as (b), but at 0.8 mTorr nitrogen gas. (e) FRPs at 0.8 mTorr nitrogen gas pressure with initial AC phase, $\alpha$, independent of RF drive phase with phase angles of $315^{\circ}$. (f) FRPs at $0.8 \mathrm{mTorr}$ nitrogen gas pressure with initial AC phase, $\alpha^{*}$, locked relative to RF drive phase.
\end{abstract}

of FRPs for prime $\beta$ values at various pressures. At 0.2 mTorr, the time between ion-nitrogen collisions is about $25 \mu \mathrm{s}$ and a typical prime trajectory period for the 609 Th ion is about $7 \mu \mathrm{s}$, so that the ion would complete about three prime trajectory periods between collisions. At the various pressures used in Table 2 with AC voltages of about $0.1 \mathrm{~V}$, we can see that significant improvements in resolution can be obtained at pressures less than $0.1 \mathrm{mTorr}$. At pressures of $0.1 \mathrm{mTorr}$ and above, reductions in frequency bandwidth of less than $4.0 \mathrm{~Hz}$ or slight increases in bandwidth are observed. These results at higher pressures indicate that collisions occur on about the same time scale as the prime trajectory period at 0.1 mTorr. We expect that any changes in the topography of the FRP at higher pressures, which may explain the reduced resolution bene- fits of prime versus ordinary $\beta$ values, are due to randomizing effect of collisions on the ion trajectories. With the exception of one value in Table 1, bandwidth reductions of greater that $10 \mathrm{~Hz}$ were obtained at $\mathrm{AC}$ voltages from 0.3 to $0.5 \mathrm{~V}$.

The frequency resolution benefits for resonant dipole excitation and ejection at prime versus ordinary $\beta$ values show the greatest potential for quadrupole cell pressures less than 0.1 mTorr. Although we speculate that the benefits of prime $\beta$ values may not be significant in Paul ion traps and nontrapping linear quadrupole collision cells, which operate at pressures above 0.1 mTorr, pressures in this range are routinely used with linear ion traps and linear quadrupole analyzers. As methodologies involving resonant excitation or ejection of ions drifting through nontrapping quadrupole cells 

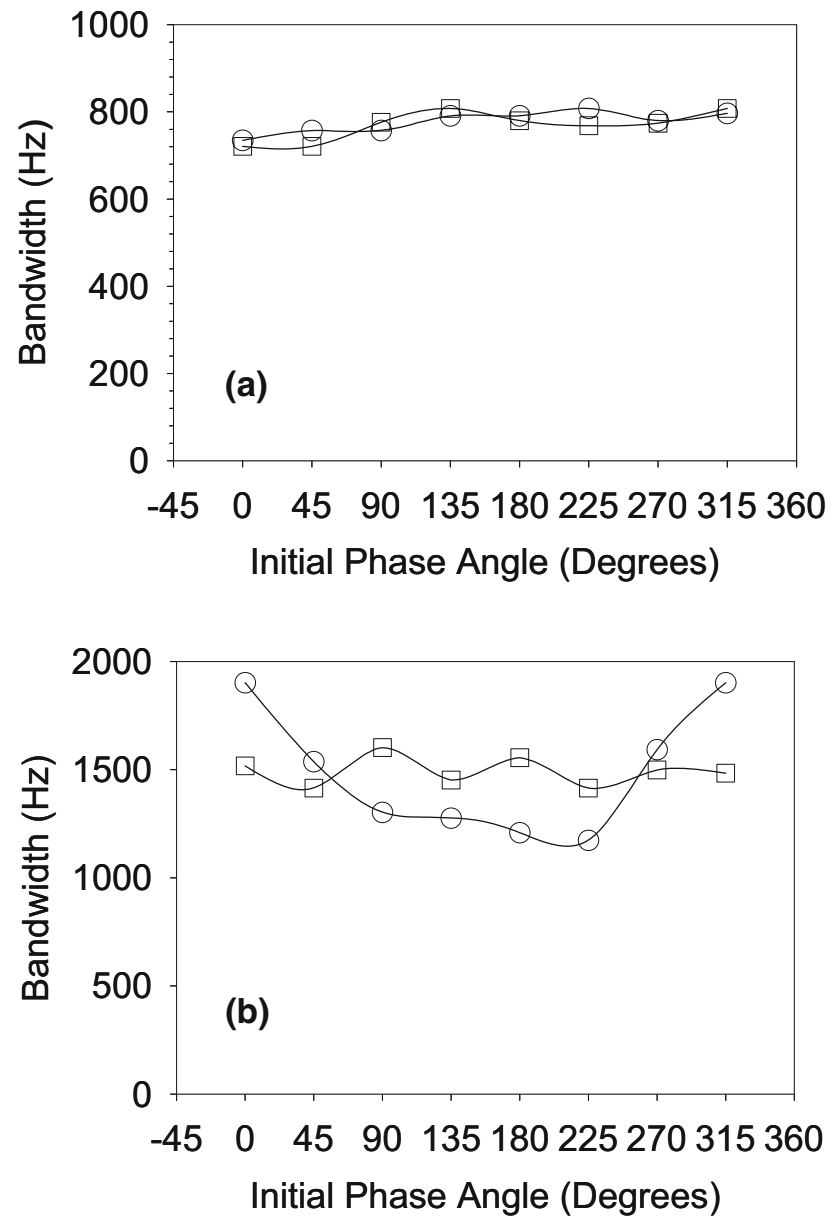

Figure 6. Variation in frequency response bandwidth at $\beta=1 / 2$ for variation in phase angle independent $(\square)$ and phase-locked $(\bigcirc)$ relative to drive RF voltage phase at (a) zero pressure and (b) 0.8 mTorr nitrogen gas in quadrupole cell.

have not found application in mass spectrometry, the results of this study have the greatest potential for linear quadrupole ion traps, which may be operated usefully at pressures below 0.1 mTorr [2]. At pressures above 0.1 mTorr, the phase of the ions trajectories and the influence of higher order fields become increasingly significant in influencing the FRP bandwidth.

Further studies in correlation between phaselocking, rod shape, and rod position with the position and magnitude of stability holes and mountains in the bandwidth versus $\beta$ relationship may produce insights that will allow for the theory presented here to be extended to the higher pressures used in Paul traps and nontrapping linear quadrupole collision cells. The phase-locking of primary interest involves setting a fixed initial phase difference between the $\mathrm{AC}$ and the $\mathrm{RF}$. The initial AC phase shift $\left(\alpha^{*}\right)$ is defined relative to a zero phase for RF. Figures 3 and 4 demonstrate the increasing influence of phase-locking on FRP resolution and peak shape with increasing pressure for $\beta=1 / 2$. As shown in Figures 5a, b, and 6a, phase-locking has little effect on either resolution or shape of the FRP at zero pressure. However, for the FRP at higher pressures, such as the case for 0.8 mTorr of nitrogen gas in the quadrupole cell presented in Figures $5 c, d$, and $6 b$, the phase-locking between AC and RF significantly affects both the profile resolution and shape. Ultimately, one is able to achieve a much greater resolution via the correct phase-locking angle, in this case $180^{\circ}<$ $\alpha^{*}<225$, than random phase or an arbitrary phase-lock of, for example, $\alpha^{*}=0$. It is, however, beyond the scope and the intent of the current study to systematically determine how optimizing phase-locking or rod shape and position can improve the benefits of prime $\beta$ values at pressures above 0.1 mTorr.

\section{Conclusions}

Prime $\beta$ values provide at least 15 to $20 \%$ improvement in frequency resolution of resonant excitation ejection of ions, but excitation voltages must be carefully tuned and collision gas pressures must be less than 0.1 mTorr. The results of frequency response profiles at varying $A C$ voltages show that Lower resonant excitation AC voltages can significantly improve resonant ejection resolution without significant loss of ejection efficiency. However, excessive gas pressure and round rods reduce average radial energy of resonant ions, resulting in reduced ejection efficiency and even failure of ejection. The combined effects of round rods and collision gas may require higher $\mathrm{AC}$ voltage to attain resonant ejection times that are short enough to be practical. The results of the present study also show that the resolution of FRPs can still benefit from prime $\beta$ values at AC voltages of up to at least $0.5 \mathrm{~V}$. Frequency interference can occur during resonant excitation even in the absence of higher-order fields; carefully choosing the $\beta$ value for resonance can minimize loss of resolution due to interference frequencies.

Many areas of study may be pursued in the future. One possible avenue of investigation is determining the reasons for interference in FRPs. Figure $5 \mathrm{e}$ and $\mathrm{f}$ show the presence of peak doublets (split peak) in FRPs at certain phase angles (most notably at $315^{\circ}$ for Figure $5 c$ and 90 degrees for 5D), while the FRPs are singlet peaks at other angles. The reason why certain phase angles produce doublets in an FRP is unknown. The origin of the "stability mountains" in bandwidth versus $\beta$ plots overlapping "stability holes" at prime $\beta$ values is also unknown. Another area of interest would be better characterization of loss of resolution enhancement with increasing pressure, such as investigating shifts in "stability holes" with increasing pressure in regions of prime $\beta$ values using bandwidth versus $\beta$ plots. Additional features that should be studied are the effects of fringing fields at the entrance and the exit of the rods and those of varying the phase-lock shift of the AC voltage with respect to the RF voltage. 


\section{Acknowledgments}

This study was funded by the Natural Sciences and Engineering Research Council (NSERC) of Canada, MDS SCIEX, and York University.

\section{References}

1. March, R. E.; Hughes, R. J.; Todd, J. F. J. Quadrupole Storage Mass Spectrometry. In Chemical Analysis: A Series of Monographs on Analytical Chemistry and Its Applications; Winefordner, J. D.; Kolthoff, I. M., Eds.; John Wiley and Sons: New York, 1989; pp 1-196.

2. Collings, B. A.; Stott, W. R.; Londry, F. A. Resonant Excitation in a Low-Pressure Linear Ion Trap. J. Am. Soc. Mass Spectrom. 2003, 14, $622-634$.

3. Campbell, J. M.; Collings, B. A.; Douglas, D. J. A New Linear Ion Trap Time-of-flight System with Tandem Mass Spectrometry Capabilities Rapid Commun. Mass Spectrom. 1998, 12, 1463-1474.

4. Collings, B. A.; Campbell, J. M.; Mao, D.; Douglas, D. J. A Combined Linear Ion Trap Time-of-Flight System with Improved Performance and MS $^{n}$ Capabilities. Rapid Commun. Mass Spectrom. 2001, 15, 1777-1795.

5. Sudakov, M.; Konenkov, N.; Douglas, D. J.; Glebova, T. Excitation Frequencies of Ions Confined in a Quadrupole Field with Quadrupole Excitation. J. Am. Soc. Mass Spectrom. 2000, 11, 10-18.

6. Sudakov, M. A Diagram of the Stable Secular Motion of Ions Trapped in an RF Quadrupole Field in the Presence of Additional Harmonic Quadrupole Excitation. Tech. Phys. Lett. 2000, 26, 870-872.

7. Konenkov, N.; Sudakov, M.; Douglas, D. J. Matrix Methods for the Calculation of Stability Diagrams in Quadrupole Mass Spectrometry. J. Am. Soc. Mass Spectrom. 2002, 13, 597-613.

8. Franzen, J. The Nonlinear Ion Trap. Part 5. Nature of Nonlinear Resonances and Resonant Ion Ejection. Int. J. Mass Spectrom. 1994, 130, $15-40$

9. Jonsson, A. P. Mass Spectrometry for Protein and Peptide Characterization. Cell. Mol. Life Sci. 2001, 58, 868-884.

10. Papayannopoulos, I. A. The Interpretation of Collision-induced Dissociation Tandem Mass Spectra of Peptides. Mass Spectrom. Rev. 1995, 14, $49-73$

11. Hunt, D. F.; Yates, J. R., III; Shabanowitz, J.; Winston, S.; Hauer, C. R. Protein Sequencing by Tandem Mass Spectrometry. Proc. Natl. Acad. Sci. U.S.A. 1986, 83, 6233-6237.

12. Smyth, W. F.; Brooks, P. A Critical Evaluation of High Performance Liquid Chromatography-Electrospray Ionization-Mass Spectrometry and Capillary Electrophoresis-Electrospray-Mass Spectrometry for the Detection and Determination of Small Molecules of Significance in Clinical and Forensic Science. Electrophoresis 2004, 25, 1413-1446.

13. Dooley, K. C. Tandem Mass Spectrometry in the Clinical Chemistry Laboratory. Clin. Biochem. 2003, 36, 471-481.

14. Maurer, H. H. Liquid Chromatography-Mass Spectrometry in Forensic and Clinical Toxicology. J. Chromatogr. B 1998, 713, 3-25.

15. Bowers, L. D. Analytical Advances in Detection of PerformanceEnhancing Compounds. Clin. Chem. 1997, 43, 1299-1304.

16. Williams, J. D.; Cox, K. A.; Cooks, R. G.; McLuckey, S. A.; Hart, K. J.; Goeringer, D. E. Resonance Ejection Ion Trap Mass Spectrometry and
Nonlinear Field Contributions: The Effect of Scan Direction on Mass Resolution. Anal. Chem. 1994, 66, 725-729.

17. Londry, F. A.; Hager, J. W. Mass Selective Axial Ejection from a Linear Quadrupole Ion Trap. J. Am. Soc. Mass Spectrom. 2003, 14, 1130-1147.

18. Sudakov, M.; Douglas, D. J. Linear Quadrupoles with Added Octopole Fields. Rapid Commun. Mass Spectrom. 2003, 17, 2290-2294.

19. Douglas, D. J.; Glebova, T. A.; Konenkov, N. V.; Sudakov, M. Y. Spatial Harmonics of the Field in a Quadrupole Mass Filter with Circular Electrodes. Tech. Phys. 1999, 44, 1215-1219.

20. Stott, W. R.; Collings, B.; Londry, F.; Hager, J. Axial Ejection Resolution in Multipole Mass Spectrometers, US Patent 20030222210A1. 2003.

21. Franzen, J. Simulation Study of an Ion Cage with Superimposed Multipole Fields. Int. J. Mass Spectrom. Ion. Processes 1991, 106, 163-178.

22. Wang, Y.; Franzen, J. The Nonlinear Resonance Ion Trap. Part 1. Potential Distribution in Hyperboloidal QUISTORs. Int. J. Mass Spectrom. Ion Processes 1992, 112, 167-178.

23. Wang, Y.; Franzen, J.; Wanczek, K. P. The Nonlinear Resonance Ion Trap. Part 2. A General Theoretical Analysis. Int. J. Mass Spectrom. Ion Processes 1993, 124, 125-144.

24. Wang, Y.; Franzen, J. The Nonlinear Resonance Ion Trap. Part 3. Multipole Components in Three Types of Practical Ion Trap. Int. J. Mass Spectrom. Ion Processes 1994, 132, 155-172.

25. Franzen, J. The Nonlinear Resonance Ion Trap. Part 4. Mass Selective Instability Scan with Multipole Superposition Int. J. Mass Spectrom. Ion Processes 1993, 125, 165-170.

26. March, R. E.; McMahon, A. W.; Londry, F. A.; Alfred, R. L.; Todd, J. F. J. Vedel, F. Resonance Excitation of Ions Stored in a Quadrupole Ion Trap. Part 1. A Simulation Study. Int. J. Mass Spectrom. Ion Processes 1989, 95, 119-156.

27. March, R. E.; McMahon, A. W.; Allinson, E. T.; Londry, F. A.; Alfred R. L.; Todd, J. F. J.; Vedel, F. Resonance Excitation of Ions Stored in a Quadrupole Ion Trap. Part II. Further Simulation Studies. Int. J. Mass Spectrom. Ion Processes 1990, 99, 109-124.

28. March, R. E.; Londry, F. A.; Alfred, R. L.; Todd, J. F. J.; Penman, A. D. Vedel, F.; Vedel, M. Resonance Excitation of Ions Stored in a Quadrupole Ion Trap. Part III. Introduction to the Field Interpolation Method. Int. J. Mass Spectrom. Ion Processes 1991, 110, 159-178.

29. Alfred, R. L.; Londry, F. A.; March, R. E.; Resonance Excitation of Ions Stored in a Quadrupole Ion Trap. Part IV. Theory of Quadrupolar Excitation. Int. J. Mass Spectrom. Ion Processes 1993, 125, 171-185.

30. Du, Z.; Douglas, D. J.; Konenkov, N. Peak Splitting with a Quadrupole Mass Filter Operated in the Second Stability Region. J. Am. Soc. Mass Spectrom. 1999, 10, 1263-1270.

31. Londry, F. A.; March, R. E. Systematic Factors Affecting High MassResolution and Accurate Mass Assignment in a Quadrupole Ion Trap. Int. J. Mass Spectrom. Ion Processes 1995, 144, 87-103.

32. Press, W. H.; Teukolsky, S. A.; Vetterling, W. T.; Flannery, B. P. Numerical Recipes in C, 2nd ed.; Cambridge University Press: London, 1992; p 724.

33. Baranov, V. I.; Bandura, D. R.; Tanner, S. D. Limitations of the Effective Potential for the Evaluation of the Ion Energy in the RF-Driven Quadrupole Field. Int. J. Mass Spectrom. 2005, 247, 40-47.

34. Abramowitz, M.; Stegun I. A. Handbook of Mathematical Functions: With Formulas, Graphs, and Mathematical Tables; Dover Publications: New York, 1972; p 727. 